Pacific Journal of Mathematics

WEAK FROBENIUS RECIPROCITY AND COMPACTNESS 


\title{
WEAK FROBENIUS RECIPROCITY AND COMPACTNESS CONDITIONS IN TOPOLOGICAL GROUPS
}

\section{ROLF WIM HENRICHS}

\begin{abstract}
We study weak containment relations between unitary representations of a locally compact group $G$ and closed subgroups $H$. We prove that certain weak Frobenius properties and compactness conditions are equivalent. Moreover, for amenable $G$ having small invariant neighborhoods at $e$ weak Frobenius reciprocity (FP) defined by Fell holds for the pair $(G, H)$ if every element of $H$ has relatively compact conjugacy class in $G$.
\end{abstract}

Introduction. In [4], Fell considers the following weak version of the Frobenius reciprocity property (FP): for every closed subgroup $H$ of a locally compact group $G$ and $\pi \in \hat{G}, \psi \in \hat{H} \pi$ is weakly contained in ${ }_{G} U^{\psi}$, the unitary representation of $G$ induced by $\psi$, if and only if $\psi$ is weakly contained in the restriction $\pi \mid H$ of $\pi$ to $H$.

Compact groups have property FP by the classical reciprocity theorem; Fell has shown that abelian groups satisfy FP.

In $\S 2$ we deal with a weaker property (RFP): reciprocity above holds for every if $\in \hat{H}$ and the trivial one dimensional representation $I_{G}$ of $G$ (not necessarily for arbitrary $\pi \in \hat{G}$ ). Property RFP is inherited by closed subgroups, we do not know whether this is true for FP. However, we have shown in [8] that for discrete groups $G$ properties FP and RFP are equivalent with $G$ to have only finite conjugacy classes. To get analogous results in the nondiscrete case we look at the normal subgroup $G_{F}$ of $G$, the union of all relatively compact conjugacy classes in $G . G_{F}$ is open if and only if there is a compact neighborhood of $e \in G$, invariant under the action of $G$ by inner automorphisms ( $G \in[\mathrm{IN}]$; see [15], for a proof). It turns out for the class of IN-groups RFP to be a compactness condition.

THEOREM A. For a locally compact group the following conditions are equivalent

(1) $G \in[I N] \cap[R F P]$

(2) $G=G_{F}$.

Also for Lie groups $G \in[\mathrm{RFP}] G_{F}$ is open as it will be shown in [3]. Thus it follows from Theorem A, that for Lie groups or connected groups $G \in \mathrm{RFP}$ is equivalent with $G$ to have only relatively compact conjugacy classes $\left(G \in[F C]^{-}\right)$.

If $G$ is an IN-group there is a compact normal subgroup $K$ of 
$G$ such that $G / K$ has small invariant neighborhoods at $e(G \in[\mathrm{SIN}])$. The results in [8] for discrete groups can be generalized to SINgroups. The following theorem shows that groups $G \in[F C]^{-} \cap[\mathrm{SIN}]$ have property FP. Combining it with Theorem A one sees that for SIN-groups RFP and FP are equivalent.

THEOREM B. Let $G$ be an amenable SIN-group. If $H$ is a closed subgroup of $G$ contained in $G_{F}$ and $\pi \in \hat{G}, \psi \in \hat{H}, \pi$ is weakly contained in ${ }_{G} U^{\psi}$ if and only if $\pi \mid H$ weakly contains $\gamma^{\prime}$.

As a corollary we get that the direct product of an abelian group and a compact group has property FP. It remains an open problem whether arbitrary $[\mathrm{FC}]^{-}$-groups have property FP. The methods used in $\S 3$ to prove the results for SIN-groups do not work in the general IN-group case.

In $\S 2$ we state some general weak containment relations for unitary representations of arbitrary locally compact groups and then prove that all conjugacy classes of an IN-group satisfying RFP have compact closure. Furthermore, we show that extensions of compact groups with groups satisfying RFP have property RFP. Therefore the proof of $2 \Rightarrow 1$ in Theorem A can be reduced to the SIN-group case.

1. Preliminaries. The following notations will be used throughout the paper:

$$
\begin{aligned}
& C^{*}(G)=C^{*} \text {-algebra of the locally compact group } G \\
& \langle,\rangle \quad=\text { canonical bilinear from on } L^{\infty}(G) \times L^{1}(G) \\
& { }_{x} f(y)=f(x y) \text { and } f_{x}(y)=f(y x) \text { for a function } f \text { on } G \\
& f^{\tau}(y)=f\left(\tau^{-1}(y)\right) \text { for an automorphism } \tau \text { of } G \\
& \operatorname{supp} f=\text { support of } f \\
& C_{00}(X)=\text { continuous functions on the locally compact space } X \\
& \text { having compact support } \\
& \operatorname{supp} \mu=\text { support of the measure } \mu \\
& \langle x\rangle \quad=\text { subgroup generated by } x \in G \\
& C(x)=\text { centralizer of } x \\
& {[G: H]=\text { index of the subgroup } H} \\
& g \mid Y=\text { restriction of a mapping } g \text { to } Y \\
& \text { ex } C=\text { set of extreme points of the convex set } C \text {. }
\end{aligned}
$$

Representation always means continuous unitary representation on a Hilbert space. $\hat{G}$ denotes the set of equivalence classes of irreducible representations of $G$. If $\pi$ is a representation of $G, \operatorname{ker} \pi$ denotes the kernel of $\pi$, considered as a representation of $C^{*}(G)$. If $S, T$ are sets of representations, we write $S \prec T$ if $S$ is weakly con- 
tained in $T$. By $[2, \S 18], S \prec T$ if and only if $\bigcap_{\pi \in S} \operatorname{ker} \pi \supseteqq \bigcap_{\pi \in T} \operatorname{ker} \pi$.

Let $P(G)$ be the set of all continuous positive definite functions on $G, P(G) \subseteq L^{\infty}(G)$ endowed with the weak *-topology. On $P^{1}(G)=$ $\{\varphi \in P(G) ; \varphi(e)=1\}$ this equals the topology of uniform convergence on compact sets in $G$, sometimes called Pontryagin topology. Every $\varphi \in P(G)$ defines a representation $\pi_{\varphi}$ of $G$ on a Hilbert space $\mathfrak{K}_{\varphi}$ with cyclic vector $\xi_{\varphi}$ such that

$$
\varphi(x)=\left(\pi_{\varphi}(x) \xi_{\varphi} \mid \xi_{\varphi}\right) \quad \text { for all } x \in G .
$$

The positive functional on $C^{*}(G)$ corresponding to $\varphi \in P(G)$ is also denoted by $\varphi, M_{\varphi}=\left\{a \in C^{*}(G) ; \varphi\left(a^{*} \alpha\right)=0\right\}$ is a left ideal in $C^{*}(G)$.

Let $N$ be a closed normal subgroup of $G$; we set $f^{x}(n)=f\left(x n x^{-1}\right)$ for a function $f$ on $N$ and $x \in G$. The extension to $C^{*}(N)$ of the mapping $f \rightarrow f^{x}$ of $C_{00}(N)$ will be written as $a \rightarrow a^{x}$. An ideal $M$ in $C^{*}(N)$ is called $G$-stable if $a \in M$ implies $a^{x} \in M$ for all $x \in G$. For a closed subgroup $H$ of $G$ we set $P(N, H)=\left\{\phi \in P(N) ; \varphi^{x}=\varnothing\right.$ for all $x \in H\} \quad$ and $P^{1}(N, H)=P(N, H) \cap P^{1}(N) . \quad P_{1}(N, H)=\{\varphi \in P(N, H)$; $\varphi(e) \leqq 1\}$ is convex and compact, $E(N, H)$ denotes the set of all nonzero extreme points of $P_{1}(N, H)$. We write $E(N)$ instead of $E(N, N)$.

Let $H$ be a closed subgroup of $G$; left Haar measures on $G$ and $H$, respectively, are denoted by $d x$ and $d s$ and let $\Delta_{G}$ and $\Delta_{I I}$ be their modular functions. For $f \in C_{00}(G)$ let $T_{H I} f \in C_{00}(G / H)$ be the function

$$
T_{H} f(\dot{x})=\int_{H} f(x s) d s, \quad x \in G .
$$

If $\psi$ is a representation of $H{ }_{G} U^{\psi}$ denotes the representation of $G$ obtained by inducing $\psi$ to $G$. For a function $f$ on $G$ we set $q(s)=$ $\left(\Delta_{G}(s) / \Delta_{H}(s)\right)^{1 / 2}$ and $R(f)=q(s) f(s), s \in H$. For $\gamma \in P(H)$ let $\mu^{r}$ be the Radon measure on $G$ defined by

$$
\mu^{r}(f)=\int_{H} \gamma(s) R(f)(s) d s, \quad f \in C_{00}(G) .
$$

By [1, Thm. 1], $\mu^{r}$ is positive definite, i.e., $\mu^{r}\left(f^{*} * f\right) \geqq 0$, let

$$
N^{r}=\left\{f \in C_{00}(G) ; \mu^{r}\left(f^{*} * f\right)=0\right\} \text { and }[f]^{r}=f+N^{r} \text {. }
$$

The completion of $C_{00}(G) / N^{r}$ with respect to the scalar product

$$
\left([f]^{r} \mid[g]^{r}\right)=\mu^{r}\left(g^{*} * f\right), \quad f, g \in C_{00}(G)
$$

is denoted by $\mathfrak{S}^{r}$. The representation ${ }_{G} U^{r}$ of $G$ on $\mathfrak{S}^{r}$ such that

$$
U_{x}^{r}[f]^{r}=\left[{ }_{x-1} f\right]^{r}, \quad f \in C_{00}(G), \quad x \in G
$$

is equivalent to ${ }_{G} U^{\pi_{\gamma}}[1]$. 
If $H$ is an open subgroup of $G$ we identify $\mathfrak{S}^{r}$ with $\mathfrak{K}_{\varphi}$ by $[f]^{r} \rightarrow \pi_{\varphi}(f) \xi_{\varphi}$, where $\varphi \in P(G)$ is the trivial extension of $\gamma, \varphi(x)=0$ for $x \in G \backslash H$.

2. Weak containment and the restricted Frobenius property RFP. If a locally compact group $G$ satisfies FP it has the following (weaker) property RFP: for every closed subgroup $H$ of $G$ and $\psi \in \hat{H}$

$$
I_{G} \prec{ }_{G} U^{\psi} \text { if and only if } \psi=I_{H} \text {. }
$$

Actually, if $\pi=I_{G}, \psi=I_{H}$ thus $\psi=\pi \mid H$, we have

$$
I_{G} \prec{ }_{G} U^{I_{H}} \quad \text { for all closed subgroups } H \text { of } G
$$

(by [6], this property is satisfied if and only if $G$ is amenable and it is equivalent to the weak Frobenius property WF1 defined by Fell in [4]: for every closed subgroup $H$ of $G$ and $\pi \in \widehat{G}$

$$
\left.\pi \prec{ }_{G} U^{\pi \mid H}\right) .
$$

Conversely, if $\psi \in \hat{H}$ and $I_{G} \prec{ }_{G} U^{\psi}$, then FP implies

$$
\psi \prec I_{H} \text { therefore } \psi=I_{H} \text {. }
$$

We do not know whether FP is inherited by closed subgroups therefore we deal with the weaker property RFP.

LEMMA 2.1. If $G$ has property RFP, closed subgroups $H$ and quotients $G / N$ have property $R F P$.

Proof.

(a) Every closed subgroup of an amenable group is amenable and by [6] satisfies WF1. The same holds for every continuous homomorphic image of $G$.

(b) Let $K$ be a closed subgroup of $H$ and let $I_{H} \prec{ }_{H} U^{\psi}, \psi \in \hat{K}$. By Theorem 4.3 in [4] and by the theorem on inducing in stages (see [18], for instance)

$$
\begin{aligned}
& { }_{G} U^{I_{H}} \prec{ }_{G} U\left({ }_{H} U^{\psi}\right)={ }_{G} U^{\psi} \text {. } \quad \text { Since } G \text { satisfies RFP } \\
& I_{G} \prec{ }_{G} U^{I_{H}} \text { and } I_{G} \prec{ }_{G} U^{\psi} \text { therefore } \psi=I_{K} \text {. }
\end{aligned}
$$

(c) Let $W$ be a closed subgroup of $G / N, N$ closed normal, and let $I_{G / N} \prec U^{\psi}, \psi=\pi_{\rho} \in \hat{W}$. Then $I_{G} \prec U^{\psi} \circ p, p: G \rightarrow G / N$ the canonical projection. If $H=p^{-1}(W)$ and $\gamma=\rho \circ p \in P^{1}(H)$, $\psi \circ p$ is the cyclic representation associated with $\gamma$. If left Haar measures of $G$ and $G / N, H$ and $W$, respectively, are normalized such that Weil's formula holds, ${ }_{G} U^{\circ \circ p}$ and ${ }_{G} U^{\psi} \circ p$ are easily seen to be equivalent: $[f]^{r} \rightarrow\left[T_{N} f\right]^{\rho}$, $f \in C_{00}(G)$, defines the corresponding intertwining operator. Therefore 
$I_{G} \prec{ }_{G} U^{\circ \circ p}$ and $\psi=I_{W}$ follows from $\psi \circ p=I_{H}$.

Let $\mu$ be a positive definite Radon measure on $G$. If $\left\{f_{i} ; i \in I\right\}$ is an approximate identity for $C_{00}(G)$ in the inductive limit topology we denote by $\pi_{i}$ the cyclic representation generated by $\pi_{\mu}$ and $\left[f_{i}\right]^{\mu}$.

LEMMA 2.2. $\pi_{\mu}$ is weakly equivalent to the set of representations $\pi_{i}, i \in I$.

Proof. Clearly $\left\{\pi_{i} ; i \in I\right\} \prec \pi_{\mu} . \quad$ Let $a \in \bigcap_{i \in I} \operatorname{ker} \pi_{i}$ and $f \in C_{00}(G)$ be given. As

$$
\left\|[f]^{\mu}-\pi_{\mu}(f)\left[f_{i}\right]^{\mu}\right\|^{2}=\mu\left(\left(f-f * f_{i}\right)^{*} *\left(f-f * f_{i}\right)\right)
$$

tends to zero and

$$
\pi_{\mu}(a) \pi_{\mu}(f)\left[f_{i}\right]^{\mu}=\pi_{i}(a) \pi_{i}(f)\left[f_{i}\right]^{\mu}=0
$$

we get $\pi_{\mu}(a)[f]^{\mu}=0 . C_{00}(G)$ being dense in $\mathfrak{S}^{\mu}$ the assertion follows.

The left regular representation of $G$ is denoted by $\lambda_{G}$, or simply $\lambda$. The crucial step exploring which groups may have RFP is the following

Proposition 2.3. Let $N$ be an open normal subgroup of $G$ and let $x$ be an element of $G$, not in $G_{F}$. Then $\lambda \prec U^{r}$ for every character $\gamma$ of $\langle x\rangle$ if one of the following conditions is satisfied

(1) $x$ has order $p, p$ prime number

(2) $x N \in(G / N)_{F}$ has infinite order and $\langle x\rangle \cap G_{F}=\{e\}$.

Proof. In both cases $\langle x\rangle$ is discrete and $\langle x\rangle \cap G_{F}=\{e\}$. Let $\gamma$ be any character of $\langle x\rangle$ and let $\left\{f_{i} ; i \in I\right\}$ be a usual approximative identity for $C_{00}(G)$ in the inductive limit topology. Since $N$ is open we may suppose $\operatorname{supp} f_{i} \subseteq N$ for $i \in I$. By Lemma 2.2, since $\lambda$ is the representation corresponding to the positive definite measure $f \rightarrow f(e)$, $f \in C_{00}(G), \lambda$ is weakly contained in the set of cyclic representations $\pi_{i}$ defined by $\lambda$ and $f_{i}, i \in I$. By [2, 18.1.4], it is sufficient to show that for every $i \in I$ the function defined by $\lambda$ and $f_{i}$ can be approximated uniformly on compact sets by positive definite functions associated with $U^{r}$. Therefore let $f \in C_{00}(G)$ with $K=\operatorname{supp} f \subseteq N$ be fixed and let $C$ be a compact set in $G$. For $c \in C, s \in\langle x\rangle, z \in G$ define

$$
g(s, c, z)=\int_{G} f\left(c^{-1} y^{-1} z^{-1} s z\right) f^{*}(y) d y
$$

Then 


$$
\begin{aligned}
\left(U_{c}^{\gamma}\left[f_{z}\right]^{\gamma} \mid\left[f_{z}\right]^{\gamma}\right)=\sum_{s \in\langle x\rangle} \gamma(s) q(s)\left(\left(f_{z}\right)^{*} * c^{-1} f_{z}\right)(s) \\
\quad=\sum_{s \in\langle x\rangle} \gamma(s) q(s) \int_{G} f\left(c^{-1} y^{-1} s z\right) \overline{f\left(y^{-1} z\right)} \Delta_{G}\left(y^{-1}\right) d y \\
\quad=\sum_{s \in\langle x\rangle} \gamma(s) q(s) g(s, c, z) \Delta_{G}\left(z^{-1}\right) .
\end{aligned}
$$

If $g(s, c, z) \neq 0 z^{-1} s z$ must be in the set $K^{-1} c K$.

Case (1). Let $|\langle x\rangle|=p$ and $k=(p-1)$ ! then $x^{k} \notin G_{F}$ and there exists $z \in G$ such that $z^{-1} x^{k} z$ is not in the compact set $\bigcup_{i=1}^{p-1}\left(K^{-1} C K\right)^{k / i}$. It follows

$$
z^{-1} x^{i} z \notin K^{-1} C K, \quad 1 \leqq i \leqq p-1
$$

therefore $g(s, c, z)=0$ if $s \neq e, c \in C$. Thus for every $c \in C$

$$
(\lambda(c) f \mid f)=\left(f^{*} *_{c^{-1}} f\right)(e)=g(e, c, z)=\left(U_{c}^{r}\left[f_{z}\right]^{r} \mid\left[f_{z}\right]^{r}\right) \Delta_{G}(z) .
$$

Case (2). We may assume that $x N$ is in the centre of $G / N$ : as $G / N$ is discrete and $[G / N: C(x N)]<\infty H=\{z \in G ; z N \in C(x N)\}$ has finite index, therefore $\langle x\rangle \cap H_{F}=\{e\}$. Then if one can prove $\lambda_{H} \prec_{H} U^{r}$ $\lambda \prec{ }_{G} U^{\lambda}{ }^{H}{ }_{G} U\left({ }_{H} U^{r}\right)={ }_{G} U^{r}$ follows.

Now if $z^{-1} s z \in K^{-1} c K \subseteq N c N$, it follows $c \in N z^{-1} s z N=s N$. Therefore $g(s, c, z)=0$ for all $s \in\langle x\rangle$ and all $z \in G$ unless $c \in\langle x\rangle N$. If $c \in N$ and $g(s, c, z) \neq 0$ then $c \in s N$ forces $s=e$ as $\langle x\rangle \cap N=\{e\}$. Thus for all $z \in G$

$$
\Delta_{G}(z)\left(U_{c}^{r}\left[f_{z}\right]^{r} \mid\left[f_{z}\right]^{r}\right)=\left\{\begin{array}{cl}
0 & c \notin\langle x\rangle N \\
(\lambda(c) f \mid f) & c \in N
\end{array}\right.
$$

Finally, there is a finite set $\left\{k_{i} ; 1 \leqq i \leqq m\right\}$ of nonzero integers such that $C \cap(\langle x\rangle N \backslash N) \subseteq \bigcup_{i=1}^{m} x^{k} i N$. As $x^{k} \notin G_{F}, k=\Pi_{i=1}^{m} k_{i}$, we may choose $z \in G$ such that

$$
z x^{k} z^{-1} \notin \bigcup_{i=1}^{m}\left(K^{-1} C K\right)^{k / k_{i}}
$$

therefore

$$
z x^{k_{i}} z^{-1} \notin K^{-1} C K \quad \text { for } 1 \leqq i \leqq m .
$$

Thus $g\left(x^{k_{i}}, c, z\right)=0$, but if $s \neq x^{k_{i}} g(s, c, z)=0$ for $c \in C \cap(\langle x\rangle N \backslash N)$ as $c \notin s N$. Consequently

$$
\left(U_{c}^{r}\left[f_{z}\right]^{r} \mid\left[f_{z}\right]^{r}\right)=0 \quad \text { for } c \in C \cap(\langle x\rangle N \backslash N) \text {. }
$$

As $(\lambda(c) f \mid f)=0$ if $c \notin N$ we have proved: there is $z \in G$ such that $(\lambda(c) f \mid f)=\Delta_{G}(z)\left(U_{c}^{r}\left[f_{z}\right]^{\gamma} \mid\left[f_{z}\right]^{r}\right)$ for all $c \in C$.

Corollary 2.4. Let $G$ be amenable and let $x \notin G_{F}$ satisfy one 
of the conditions in Proposition 2.3. Then for every $\gamma \in\langle\hat{x}\rangle$ the representation $U^{r}$ of $C^{*}(G)$ is faithful (ker $U^{r}=0$ ).

CoROLlaRY 2.5. If $G$ has property RFP every element of finite order belongs to $G_{F}$.

Proof. If not, let $n$ be the smallest number $n \in N$ for which there exist a group $H \in[\mathrm{RFP}]$ and $x \in H \backslash H_{F}$ of order $n$. Then $n$ cannot be a prime number. Otherwise there would exist a character $\gamma$ of $\langle x\rangle, \gamma \neq \equiv 1$, such that $I_{H} \prec U^{r}$ in contrary to $H \in[R F P]$. If $n=m r, n \neq m, r \in N, x^{m} \in H_{F}$ as $n$ is minimal. By [7, Thm. 3.11], there is a compact normal subgroup $K$ of $H$ with $x^{m} \in K$. As $H / K \in$ [RFP] and $|\langle x K\rangle|<n$

$x K \in(H / K)_{F}$ therefore $x \in H_{F}$, a contradiction.

For example, the euclidean group of the plane cannot have property RFP by Corollary 2.5.

LEMma 2.6. Let $G$ satisfy RFP and let $\langle x\rangle$ be isomorphic to Z. Then $x \in C\left(x^{n}\right)_{F}$ for all $n \in N$.

Proof. By Lemma 2.1, the group $H=C\left(x^{n}\right) /\left\langle x^{n}\right\rangle$ has RFP and $x\left\langle x^{n}\right\rangle \in H_{F}$ follows from the last corollary. Let $k$ be compact such that

$$
\left\{y x y^{-1} ; y \in C\left(x^{n}\right)\right\} \subseteq K\left\langle x^{n}\right\rangle \subseteq G .
$$

If $y x y^{-1}=k x^{n m(y)}, k \in K, m(y) \in Z$, it follows

$$
x^{n}=k^{n} x^{n^{2} m(y)} \quad \text { as } \quad y \in C\left(x^{n}\right) .
$$

Thus $x^{n-n^{2} m(y)}$ belongs to the finite set $K^{n} \cap\left\langle x^{n}\right\rangle$. Therefore there is a finite set $M \subseteq Z$ such that

$$
\left\{y x y^{-1} ; y \in C\left(x^{n}\right)\right\} \leqq\left\{k x^{n m} ; k \in K, m \in M\right\}
$$

which proves the lemma.

If $V$ is a normal vector group in $G$ and $x \in G_{F} x v x^{-1} v^{-1}$ is a compact element of $V$ for every $v \in V$ so that $V \subseteq C(x)[7,(3.4)]$. Now we can prove

THEOREM 2.7. If $G \in[I N]$ has property RFP then all conjugacy classes in $G$ have compact closure.

Proof.

(a) First let $G$ be discrete and let $x G_{F} \in\left(G / G_{F}\right)_{F}$. By Proposition 
2.3, (2) there exists $n \in N$ with $x^{n} \in G_{F}$ (take $N=G_{F}$ ). If $\langle x\rangle$ is not finite, $x \in C\left(x^{n}\right)_{F}$ by Lemma 2.6 thus $x \in G_{F}$ as $\left[G: C\left(x^{n}\right)\right]<\infty$, and if $\langle x\rangle$ is finite $x \in G_{F}$ by Corollary 2.5. Therefore $\left(G / G_{F}\right)_{F}$ consists of one element so that $G=G_{F}$ by Lemma 2 in [8].

(b) Let $G \in[I N] \cap[R F P]$, we may assume $G \in[S I N]$. By [22], there exists a compact normal subgroup $K$ of $G$ and closed normal subgroups $V, D$ of $G / K, V$ a vector group and $D$ discrete, such that $(G / K)_{F}$ is the direct product of $V$ and $D^{1}$. Again we may assume $K=\{e\}$. As $G_{F}$ is open $G / G_{F}=\left(G / G_{F}\right)_{F}$ by (1a), and Proposition 2.3 shows that for every element $x$ in $G$ there exists $n \in N$ with $x^{n} \in G_{F}$.

If the closed subgroup generated by $x$ is compact, $x^{n}$ is compact in $G_{F}$ and by [7, Thm. 3.11] $x^{n}$ generates a compact normal subgroup $K$ of $G$. As $x K$ has finite order $x \in G_{F}$, therefore $V \subseteq C(x)$. If $\langle x\rangle \cong Z, x \in C\left(x^{n}\right)_{F}$ and again $V \cong C(x)$ as $V \cong C\left(x^{n}\right)$. Thus $V$ is contained in the centre of $G$.

If for $x \in G x^{n}=v d, v \in V, d \in D$ we have $C(d) \leqq C\left(x^{n}\right)$. As $d$ belongs to a finite conjugacy class $\left[G: C\left(x^{n}\right)\right]<\infty$ and as $x \in C\left(x^{n}\right)_{F}$ $x \in G_{F}$ follows.

It is an interesting question whether groups $G \notin[\mathrm{IN}]$ can have property RFP. It will be shown in [3] that every Lie group or connected group $G \in[\mathrm{RFP}]$ is an IN-group. Now let $H$ be a closed subgroup of an arbitrary locally compact group $G, \pi \in \hat{G}, \psi \in \hat{H}$. If $K$ is compact normal and $\psi(H \cap K)=\{I\}$

$$
\dot{\psi}(\dot{s})=\psi(s), \quad s \in H
$$

defines a continuous irreducible representation $\dot{\psi}$ of the closed subgroup $H K / K$ in $G / K$.

Proposition 2.8. Let $\pi \in \hat{G}$ and let $K$ be a compact normal subgroup of $G$ such that $\pi(K)=\{I\}$. If $\pi \prec U^{\psi}$ for $\psi \in \hat{H}$ then $\psi(H \cap K)$ $=\{I\}$ and $\dot{\pi} \prec U \dot{\psi}$.

Proof. Let $\pi=\pi_{\varphi}$ and $\psi=\pi_{\gamma}, \varphi \in P^{1}(G), \gamma \in P^{1}(H)$. For $f \in C_{00}(G)$ define ${ }^{K} f \in C_{00}(G)$ by

$$
{ }^{k} f(x)=\int_{K} f(k x) d x, x \in G \text { where } d k \text { denotes the }
$$

normalized Haar measure on $K$. As ${ }^{K} f(x k y)={ }^{K} f(x y)$ for all $k \in K$,

${ }^{1}$ I am indebted to the referee for pointing out that the proof in [22] contains an error (in the proof, on the fourth line of p. 328, that $L$ is $B$-invariant) and for giving a sketch of how to correct that error: it suffices to prove that when $W \times D$ is in $[F C]_{B}$ with $W \sim \boldsymbol{R}^{n}$ and $D$ discrete abelian, then $W$ has a $B$-invariant complement $D_{1}$. Observing first that $G=W \times D$ is also in $[\mathrm{SIN}]_{B}$ since $W$ is characteristic and open, one can then apply a splitting theorem of Hofmann and Mostert to $\hat{G}=\hat{W} \times \hat{D}$ to find a $B$-invariant complement $\hat{W}_{1}$ to $\hat{D}$. Then take $D_{1}=\hat{W}_{1}^{\perp}$. 
$x, y \in G$, an easy computation shows

$$
\int_{K}\left(U_{x k-1}^{\gamma}[f]^{\gamma} \mid[f]^{\gamma}\right) d k=\left(U_{x}^{\gamma}\left[{ }^{K} f\right]^{\gamma} \mid\left[{ }^{k} f\right]^{\gamma}\right)
$$

Now let a compact set $C \subseteq G / K$ and $\varepsilon>0$ be given. $C_{00}(G)$ being dense in $\mathfrak{S}^{r}$ it follows from $\pi \prec U^{\psi}$ that there exist $f_{i} \in C_{00}(G)$, $1 \leqq i \leqq m$, such that

$$
\left|\varphi\left(x k^{-1}\right)-\sum_{i=1}^{m}\left(U_{x k^{-1}}^{r}\left[f_{i}\right]^{r} \mid\left[f_{i}\right]^{r}\right)\right| \leqq \varepsilon, \quad \text { for } k \in K, x \in p^{-1}(C),
$$

$p: G \rightarrow G / K$ the canonical projection. Since $\varphi\left(x k^{-1}\right)=\varphi(x), k \in K$, and using (2.1) we get

$$
\left|\varphi(x)-\sum_{i=1}^{m}\left(U_{x}^{\gamma}\left[{ }^{K} f_{i}\right]^{\gamma} \mid\left[{ }^{K} f_{i}\right]^{\gamma}\right)\right| \leqq \varepsilon, \quad x \in p^{-1}(c) .
$$

At first, we conclude from (2.2) that there exists a function $f \in C_{00}(G)$ such that $\left[{ }^{K} f\right]^{\gamma} \neq 0$, let $\left\|\left[^{K} f\right]^{r}\right\|=1$. By Blattner's theorem (see [18, Thm. 4.4]), $R\left(\left({ }^{K} f\right)^{*}{ }^{k} f\right)$ is a positive element of $C^{*}(H)$, let $T=\left(R\left(\left({ }^{K} f\right)^{*}{ }^{k} f\right)\right)^{1 / 2}$. Then for $k \in H \cap K$

$$
\begin{aligned}
\psi(k) \psi\left(T^{2}\right) & =\int_{H} q\left(k^{-1} s\right)\left(\left({ }^{K} f\right)^{*}{ }^{K} f\right)\left(k^{-1} s\right) \psi(s) d s \\
& =\int_{H} q(s)\left(\left({ }^{K} f\right) *{ }^{K} f\right)(s) \psi(s) d s=\psi\left(T^{2}\right)=\psi\left(T^{2}\right) \psi(k)
\end{aligned}
$$

therefore $\psi(T)$ commutes with $\psi(k)$ and for all $k \in H \cap K$

$$
\begin{aligned}
& \left(\psi(k) \psi(T) \xi_{\gamma} \mid \psi(T) \xi_{\gamma}\right)=\left(\psi\left(T^{2}\right) \xi_{\gamma} \mid \xi_{\gamma}\right) \\
& \quad=\int_{H} R\left(\left({ }^{K} f\right){ }^{*}{ }^{K} f\right)(s) \gamma(s) d s=\left\|\left[{ }^{K} f\right]^{\gamma}\right\|^{2}=1 .
\end{aligned}
$$

But then $\left\|\psi(k) \psi(T) \xi_{\gamma}-\psi(T) \xi_{\gamma}\right\|^{2}=0$ thus

$$
\psi(k) \psi(s) \psi(T) \xi_{\gamma}=\psi(s) \psi\left(s^{-1} k s\right) \psi(T) \xi_{\gamma}=\psi(s) \psi(T) \xi_{\gamma}
$$

for all $s \in H$. Since $\psi$ is irreducible and $\psi(T) \xi_{\gamma} \neq 0$

$$
\psi(k)=I \quad \text { for all } k \in H \cap K \text {. }
$$

If Haar measures on $G / K$ and $H K / K$, respectively, are suitable chosen and if $\rho \in P^{1}(H K / K)$ is defined by $\rho(p(s))=\gamma(s), s \in H$, it is easy to see that

$$
\left(U_{p(x)}^{\rho}\left[T_{K} f_{i}\right]^{\rho} \mid\left[T_{K} f_{i}\right]^{\rho}\right)=\left(U_{x}^{\gamma}\left[{ }^{K} f_{i}\right]^{\gamma} \mid\left[{ }^{K} f_{i}\right]^{\gamma}\right), \quad x \in G
$$

therefore (2.2) shows $\dot{\pi} \prec U \dot{\psi}$.

COROLlaRy 2.9. If $G$ is an extension of a compact group $K$ 
with a group satisfying RFP, G has property RFP.

Proof. $G$ is amenable, if $G / K$ is amenable, $K$ compact, normal. If $\psi \in \hat{H}$ is such that $I_{G} \prec{ }_{G} U \psi, I_{G / K} \prec U \psi \dot{\psi}$ holds by the proposition. $G / K \in[\mathrm{RFP}]$ implies $\dot{\psi}=I_{H K / K}$ thus $\psi=I_{H}$.

If $\phi \in P(G)$

$$
\left\langle\varphi^{x} \mid H, h\right\rangle=\int_{H} h(s)\left(\pi_{\varphi}(s) \pi_{\varphi}(x) \xi_{\varphi} \mid \pi_{\varphi}(x) \xi_{\varphi}\right) d s
$$

for $h \in L^{1}(H), x \in G$. Thus for $a \in C^{*}(H), x \in G$

$$
\left(\varphi^{x} \mid H\right)(a)=\left(\left(\pi_{\varphi} \mid H\right)(a) \pi_{\varphi}(x) \xi_{\varphi} \mid \pi_{\varphi}(x) \xi_{\varphi}\right)
$$

so that $a \in M_{\varphi x_{\mid H}}$ if and only if $\left(\pi_{\varphi} \mid H\right)(a) \pi_{\varphi}(x) \xi_{\varphi}=0$. Since $\xi_{\varphi}$ is cyclic for $\pi_{\varphi}$ we get a characterization of $\operatorname{ker} \pi_{\varphi} \mid H$ by left ideals corresponding to positive definite functions on $H$

$$
\operatorname{ker} \pi_{\varphi} \mid H=\bigcap_{x \in G} M_{\varphi x_{\mid H}} \text {. }
$$

If $\varphi$ is a class function on $G$

$$
\operatorname{ker} \pi_{\varphi} \mid H=M_{\varphi \mid H}=\operatorname{ker} \pi_{\varphi \mid H} .
$$

We shall make frequent use of these formulas. We apply (2.3) ito prove the following lemma which will be used in $\S 3$.

LEMMA 2.10. Let $H$ be a closed subgroup of a locally compact group G. Then $\pi_{\varphi} \prec{ }_{G} U^{\varphi \mid H}$ for $\phi \in P(G)$ if either

\section{$G / H$ has finite volume or}

$H$ is normal and $G / H$ is amenable.

Proof. First let $H$ be a normal subgroup of $G, G / H$ amenable. By (2.3) we have

$$
\begin{aligned}
\operatorname{ker} \pi_{\varphi} \mid H & =\bigcap_{x \in G} M_{(\varphi \mid I)^{x}}=\bigcap_{x \in G} \bigcap_{s \in H} M_{((\varphi \mid H) x)^{s}} \\
& =\bigcap_{x \in G} \operatorname{ker} \pi_{(\varphi \mid H)^{x}}
\end{aligned}
$$

therefore $\pi_{\varphi} \mid H$ is weakly equivalent to the set of representations $\left(\pi_{\varphi \mid H}\right)^{x}, x \in G$. Since the representations induced by $\left(\pi_{\varphi \mid H}\right)^{x}, x \in G$, are equivalent to ${ }_{G} U^{\varphi \mid H}$

$$
{ }_{G} U^{\pi \varphi \mid H} \prec{ }_{G} U^{\varphi \mid H} \text {, and } \pi_{\varphi} \prec{ }_{G} U^{\pi_{\varphi} \mid H} \text { as } G / H \text { is amenable [6]. }
$$

Now let $G / H$ have finite volume. We state 


$$
\left\|[f]^{\varphi}\right\|^{2} \leqq \nu(G / H)\left\|[f]^{r}\right\|^{2}, \quad f \in C_{00}(G)
$$

where $\nu$ is an invariant measure on $G / H$ and $\gamma=\varphi \mid H$ : considering $\pi_{\gamma}$ as a subrepresentation of $\pi_{\varphi} \mid H$ and using the fact that $\Delta_{G}$ and $\Delta_{H}$ coincide on $H$ it is easy to check

$$
\begin{aligned}
\left\|[f]^{\varphi}\right\|^{2} & =\int_{G} \int_{G} \varphi\left(y^{-1} x\right) f(x) \overline{f(y)} d y d x \\
& =\int_{G} \int_{G} b(x) b(y)\left(\pi_{\varphi}(x) \pi_{\gamma}\left(R\left(_{x} f\right)\right) \xi_{r} \mid \pi_{\varphi}(y) \pi_{\gamma}\left(R\left(_{y} f\right)\right) \xi_{r}\right) d y d x
\end{aligned}
$$

where $b$ denotes a Bruhat function for $H$. Therefore

$$
\begin{aligned}
\left\|[f]^{\varphi}\right\| & \leqq \int_{G} b(x)\left\|\pi_{\varphi}(x) \pi_{r}\left(R\left(_{x} f\right)\right) \xi_{r}\right\| d x \\
& =\int_{G / H} \int_{H} b(x s)\left\|\pi_{\gamma}\left(R\left(_{x s} f\right)\right) \xi_{r}\right\| d s d \nu(\dot{x}) .
\end{aligned}
$$

Since the function $\left.x \rightarrow \| \pi_{\gamma}\left(R{ }_{x} f\right)\right) \xi_{\gamma} \|$ is constant on cosets (as $q(s)=$ $1, s \in H)$ and $\int_{H} b(x s) d s=1, x \in G$

$$
\begin{aligned}
\left\|[f]^{\varphi}\right\|^{2} & \leqq\left(\int_{G / H}\left\|\pi_{\gamma}\left(R\left(_{x} f\right)\right) \xi_{r}\right\| d \nu(\dot{x})\right)^{2} \\
& \leqq \nu(G / H) \int_{G / H}\left\|\pi_{\gamma}\left(R\left(_{x} f\right)\right) \xi_{\gamma}\right\|^{2} d \nu(\dot{x}) \\
& =\nu(G / H) \int_{G} b(x)\left\|\pi_{\gamma}\left(R\left(_{x} f\right)\right) \xi_{\gamma}\right\|^{2} d x
\end{aligned}
$$

but

$$
\int_{G} b(x)\left\|\pi_{r}\left(R\left(_{x} f\right)\right) \xi_{r}\right\|^{2} d x=\left\|[f]^{r}\right\|^{2}
$$

by Blattner's theorem (see [18, Thm. 4.4]). Now let $\left\{f_{i}, i \in I\right\}$ be an approximate identity for $C_{00}(G)$ in the inductive limit topology and for $i \in I$ let

$$
\begin{aligned}
\varphi_{i}(x) & =\left(\pi_{\varphi}(x)\left[f_{i}\right]^{\varphi} \mid\left[f_{i}\right]^{\varphi}\right), \\
\rho_{i}(x) & =\left(U_{x}^{\gamma}\left[f_{i}\right]^{\gamma},\left[f_{i}\right]^{\gamma}\right), \quad x \in G .
\end{aligned}
$$

Then for $f \in C_{00}(G)$

$$
\varphi_{i}\left(f^{*} * f\right)=\left\|\left[f * f_{i}\right]^{\varphi}\right\|^{2} \leqq \nu(G / H) \rho_{i}\left(f^{*} * f\right)
$$

thus $\pi_{\varphi_{i}}$ is a subrepresentation of $\pi_{\rho_{i}}$ by $[2,2.5 .1]$. Since $\pi_{\rho_{i}}$ is contained in $U^{r}$ and $\pi_{\varphi} \prec\left\{\pi_{\varphi_{i}}, i \in I\right\}$ (by Lemma 2.2) $\pi_{\varphi} \prec U^{r}$.

REMARK 2.11. If $G$ is first countable we can choose $r_{i}>0$, $i \in N$, such that $f_{0}=\sum_{i \in N} r_{i} f_{i}^{*} * f_{i} \in C_{00}(G)$. Then one shows as in [11] 
that $\left[f_{0}\right]^{\varphi}$ is a cyclic vector for $\pi_{\varphi}$ (the lemma used in [11] is correct if the measure is defined by a positive definite function). Therefore $\pi_{\varphi}$ is a subrepresentation of $U^{r}$ in the case $G / H$ to have finite volume.

COROLLARY 2.12. Let $G=G_{n+1}$ be amenable and let $G_{i}, 1 \leqq i$ $\leqq n$, be an ascending chain of closed subgroups of $G$. If $G_{i}$ is normal in $G_{i+1}$ or if $G_{i+1} / G_{i}$ has finite volume, $1 \leqq i \leqq n$, then $\pi_{\varphi} \prec{ }_{G} U^{\varphi \mid G_{1}}$ for all $\varphi \in P(G)$.

Proof. Let $\rho=\varphi \mid G_{n}$ and suppose

$$
\pi_{\rho} \prec{ }_{G_{n}} U^{\rho \mid G_{1}}
$$

then

$$
{ }_{G} U^{\rho} \prec{ }_{G} U\left({ }_{G_{n}} U^{\rho \mid G_{1}}\right)={ }_{G} U^{\varphi \mid G_{1}} .
$$

Using Lemma 2.10 the assertion follows by induction.

By Corollary 2.9, in order to prove that groups $G \in[F C]^{-}$have RFP we may suppose $G \in[\mathrm{SIN}]$.

3. Topological Frobenius properties for SIN-groups. Let $H$ be a closed subgroup of a SIN-group $G$ and $\psi$ be a unitary representation of $H$. It has been shown in [9] that the restriction to $H$ of ${ }_{G} U^{\psi}$ contains $\psi$ as a subrepresentation therefore

THEOREM 3.1. SIN-groups have property WF2 (defined by Fell in [4]: for every closed subgroup $H$ and $\left.\psi \in \hat{H} \psi \prec{ }_{G} U^{\psi} \mid H\right)$.

Representations corresponding to positive definite measures of metric groups are known to be cyclic. What we shall need is the following fact.

Proposition 3.2. Let $G \in[\mathrm{SIN}]$ be first countable. If $\gamma \in P^{1}(H)$ is indecomposable then there exists an extension $\varphi \in P(G)$ of $\gamma$ such that $\pi_{\varphi}$ is weakly equivalent to ${ }_{G} U^{r}$.

Proof. As $G \in[\mathrm{SIN}]$ there is an approximate identity for $C_{00}(G)$ in the inductive limit topology consisting of class functions (see [7] or [9]). Moreover, we can choose $f_{i} \in C_{00}(G)$ and $r_{i}>0$ such that supports $S_{i}$ of $f_{i}^{*} * f_{i}$ are contained in a compact set $K$ and $g_{n}=$ $\sum_{i=1}^{n} r_{i} f_{i}^{*} * f_{i}$ converges uniformly on $K$ to a class function $f \in C_{00}(G)$. Since $f_{i}$ is a class function for $x \in G$

$$
\begin{aligned}
\rho_{i}(x): & =\left(U_{x}^{\gamma}\left[f_{i}\right]^{r} \mid\left[f_{i}\right]^{\gamma}\right)=\mu^{r}\left(f_{i}^{*} *{ }_{x^{-1}} f_{i}\right) \\
& =\mu^{r}\left(\left(f_{i}^{*} * f_{i}\right)_{x^{-1}}\right) .
\end{aligned}
$$


We define

$$
\varphi(x)=\mu^{r}\left(f_{x^{-1}}\right), \quad x \in G
$$

then $\varphi$ is continuous as $x \rightarrow f_{x^{-1}}$ is continuous and $\mu^{r}$ is a Radon measure. Furthermore, $\varphi$ is positive definite as

$$
\varphi(x)=\lim _{n \rightarrow \infty} \sum_{i=1}^{n} r_{i} \rho_{i}(x) \quad \text { for } x \in G .
$$

By Lemma 2.1 in [9] $\rho_{i} \mid H=\rho_{i}(e) \gamma$ and by the proof of that lemma we may assume $\mu^{r}(f)=1$ therefore

$$
\varphi \mid H=\gamma \sum_{i=1}^{\infty} r_{i} \rho_{i}(e)=\gamma \sum_{i=1}^{\infty} r_{i} \mu^{\gamma}\left(f_{i}^{*} * f_{i}\right)=\gamma .
$$

Now let $g \in C_{00}(G), S=\operatorname{supp} g$ then

$$
\begin{gathered}
\left|\langle\varphi, g\rangle-\sum_{i=1}^{n} r_{i}\left\langle\rho_{i}, g\right\rangle\right| \leqq \int_{S}|g(x)|\left|\mu^{\gamma}\left(\left(f-g_{n}\right)_{x^{-1}}\right)\right| d x \\
\leqq \int_{S}|g(x)| \int_{H}|\gamma(s)|\left|\left(f-g_{n}\right)\left(s x^{-1}\right)\right| d s d x \\
\quad \leqq \sup _{y \in K}\left|\left(f-g_{n}\right)(y)\right| \cdot \int_{H \cap K S} d s \cdot\|g\|_{L^{1}(G)}
\end{gathered}
$$

hence for all $a \in C^{*}(G)$

$$
\varphi(a)=\sum_{i=1}^{\infty} r_{i} \rho_{i}(a) \text {. }
$$

Since $\varphi^{x}(a)=\varphi\left(a^{x^{-1}}\right), x \in G$, by $[17,1.8]$,

$$
\varphi^{x}(a)=\sum_{i=1}^{\infty} r_{i} \rho_{i}^{x}(a) \quad \text { for } a \in C^{*}(G), x \in G .
$$

As $r_{i}>0 \varphi^{x}\left(a^{*} a\right)=0$ if and only if $\rho_{i}^{x}\left(a^{*} a\right)=0$ for $i \in N$ thus

$$
\operatorname{ker} \pi_{\varphi}=\bigcap_{x \in G} M_{\varphi x}=\bigcap_{i \in N} \operatorname{ker} \pi_{\rho i} .
$$

By Lemma 2.2, $U^{r}$ is weakly equivalent to $\left\{\pi_{\rho_{i}}, i \in N\right\}$ hence $U^{r}$ and $\pi_{\varphi}$ are weakly equivalent.

Let $N$ be a closed normal subgroup of $G \in[\mathrm{SIN}]$ contained in $G_{F}$ and let $\operatorname{Aut}(N)$ be the group of all topological automorphisms of $N$ with the Birkhoff topology [10, $\S 26] . \quad I(N, H)$ denotes the subgroup of all $n \rightarrow x n x^{-1}$, for $x$ in a closed subgroup $H$ of $G$, then $B \equiv \overline{I(N, H)}$ is compact in Aut $(N)$ [7, Thm. (0.1)] and we define as in [17]:

$f^{H}(n)=\int_{B} f^{\tau}(n) d \tau$ where $d \tau$ is the normalized Haar measure on $B$. If $\rho \in P(N) \rho^{H} \in P(N, H)$ and $\rho \rightarrow \rho^{H}$ is a continuous affine mapping from $P_{1}(N)$ onto $P_{1}(N, H)[17,1.9]$. 
Furthermore, for $a \in C^{*}(N)$

$$
\rho^{H}(a)=\int_{B} \rho^{*}(a) d \tau \text {. }
$$

Since $\tau \rightarrow \rho^{\tau}(\alpha)$ is continuous on $B$

$$
M_{\rho H}=\bigcap_{\tau \in B} M_{\rho} \tau=\bigcap_{x \in H} M_{\rho x}
$$

combining this with (2.3) we get for $\varphi \in P(G)$

$$
\operatorname{ker}\left(\pi_{\varphi} \mid N\right)=M_{(\varphi \mid N)^{G}}=\operatorname{ker} \pi_{(\varphi \mid N)^{G}} .
$$

If $\varphi \in P^{1}(G)$ is associated with $\pi \in \hat{G},(\varphi \mid N)^{G} \in E(N, G)$ by Lemma 1 in [13]. Conversely, if $\alpha \in E(N, G)$ we can find an indecomposable function $\rho \in P^{1}(N)$ satisfying $\rho^{\sigma}=\alpha$. By [9, Satz 2] there exists an extension $\varphi \in \operatorname{ex} P^{1}(G)$ of $\rho$, thus $(\varphi \mid N)^{G}=\alpha$. The mapping $\varphi \rightarrow$ $(\varphi \mid N)^{G}, \varphi \in \operatorname{ex} P^{1}(G)$, is continuous and $\alpha \rightarrow M_{\alpha}$ defines a homeomorphism of $E(N, G)$ onto $G-\operatorname{Max} C^{*}(N)$ the set of all maximal modular $G$-stable ideals of $C^{*}(N)$ endowed with hull-kernel topology [17, Proposition 4.8]. Therefore

Proposition 3.3. $\pi \rightarrow \operatorname{ker}(\pi \mid N)$ defines a continuous map from $\widehat{G}$ onto $G-\operatorname{Max} C^{*}(N)$.

REMARK 3.4. If $N$ is open we can consider $C^{*}(N)$ as a subalgebra of $C^{*}(G)$ thus $\operatorname{ker}(\pi \mid N)=\operatorname{ker} \pi \cap C^{*}(N)$. In this case the $\operatorname{map} \pi \rightarrow \operatorname{ker}(\pi \mid N)$ has been studied in [13] and has some more properties stated in [13, Thm. 1].

Let $H$ be a closed subgroup of $G$ and $\rho \in E(N, H)$. Since $P_{1}(N)$ is compact, convex there exists $\varphi \in \operatorname{ex} P_{1}(N)$ satisfying $\varphi^{H}=\rho$. By changing order of integration, for $n \in N$

$$
\begin{aligned}
\rho^{G}(n) & =\int_{\overline{I(N, G)}} \phi^{H}\left(\tau^{-1}(n)\right) d \tau=\int_{\overline{I(N, H)}}\left(\int_{\overline{I(N, G)}} \phi^{\tau \sigma}(n) d \tau\right) d \sigma \\
& =\varphi^{G}(n) \quad \text { thus } \rho^{G}=\phi^{G} \in E(N, G)[17,5.1] .
\end{aligned}
$$

In the following lemma we summarize such functorial properties and further known facts concerning $E(N, H)$ used in this paper.

LEMMA 3.5. Let $H$ be a closed subgroup of $G \in[\mathrm{SIN}]$ and let $N$ be a closed normal subgroup of $G$ contained in $G_{F}$.

(1) $\varphi \rightarrow \varphi \mid H$ maps $E(G, H)$ onto $E(H)[9$, Lemma 1.3 and Satz $2]^{2}$.

${ }^{2}$ Lemma 1.3 in [9] holds for arbitrary locally compact groups. The notation $I(H)$ in [9] does not refer to the inner automorphisms of $H$ but rather to the inner automorphisms of $G$ induced by elements of $H$. 
(2) $\varphi \rightarrow(\varphi \mid N)^{G}$ maps ex $P^{1}(G)$ onto $E(N, G)$.

(3) If $\rho \in E(N, H), \rho^{G}$ is in $E(N, G)$.

(4) The closure $F(N, H)$ of $E(N, H)$ with respect to the Pontryagin topology is locally compact and $F(N, H) \cup\{0\}$ is equal to the weak *-closure of ex $P_{1}(N, H)=E(N, H) \cup\{0\}$ [9, Korollar 2.8].

(5) If $N$ is contained in $H$, ex $P_{1}(N, H)$ is compact [17, 4.2; 12, Satz 1; 21, Satz 1].

Let $N$ be contained in $H$. Then it is well known that for given $\beta \in P^{1}(N, H)$ there exists a unique normalized positive Radon measure $\mu$ on $P_{1}(N, H)$ such that $\mu$ has resultant $\beta$, i.e.,

$$
\langle\beta, f\rangle=\int_{\Gamma_{1}(N, H)}\langle\gamma, f\rangle d \mu(\gamma) \quad \text { for all } f \in L^{1}(N),
$$

and supp $\mu \subseteq \operatorname{ex} P_{1}(N, H)$ holds [20, Satz 1; 17, 2.2]. If $N=H$ the unique measure $\mu$ is denoted by $\mu_{\beta}$. For arbitrary subgroups $H$ of $G$ maximal measures on $P_{1}(N, H)$ (with respect to Choquet ordering) having resultant $\beta$ don't need to be unique.

LEMMA 3.6. Let $N$ be a closed normal subgroup of $G \in$ [SIN] contained in $G_{F}$ and for $\beta \in P^{1}(N, G)$ let $\mu$ be the unique maximal measure on $P_{1}(N, G)$ with resultant $r(\mu)=\beta$.

(1) If $H$ is a closed subgroup of $G$ and if $\nu$ is any maximal measure on $P_{1}(N, H)$ such that $r(\nu)^{G}=\beta$ then

$$
\operatorname{supp} \mu=(\operatorname{supp} \nu)^{G}=\left\{\rho^{G} ; \rho \in \operatorname{supp} \nu\right\} .
$$

(2) For $\alpha \in E(N, G)$

$$
\pi_{\alpha} \prec \pi_{\beta} \text { if and only if } \alpha \in \operatorname{supp} \mu .
$$

Proof.

(1) The image $\nu^{G}$ of $\nu$ corresponding to the continuous affine mapping $\rho \rightarrow \rho^{G}$ from $P_{1}(N, H)$ onto $P_{1}(N, G)$ has resultant $r(\nu)^{G}=\beta$ and

$$
\operatorname{supp} \nu^{G}=(\operatorname{supp} \nu)^{G} \cong\left(\overline{\operatorname{ex} P_{1}(N, H)}\right)^{G} \subseteq E(N, G) \cup\{0\}
$$

(this follows from Choquet theory and Lemma 3.5). By uniqueness $\mu=\nu^{G}$ and the assertion follows.

(2) Since $\mu$ has resultant $\beta$

$$
\beta(a)=\int_{P_{1}(N, G)} \gamma(a) d \mu(\gamma) \quad \text { holds for } a \in C^{*}(N)
$$

thus

$$
M_{\beta}=\bigcap_{\gamma \in \operatorname{supp} \mu} M_{\gamma}=\bigcap_{0 \neq \gamma \in \operatorname{supp} \mu} M_{\gamma}
$$


as $\gamma \rightarrow \gamma(a)$ is continuous on $P_{1}(N, G)$ for every $a \in C^{*}(N)$. Since $\alpha, \beta$ are class functions $\operatorname{ker} \pi_{\alpha}=M_{\alpha} \supseteqq M_{\beta}=\operatorname{ker} \pi_{\beta}$ if $\alpha \in \operatorname{supp} \mu$. Conversely, if $\pi_{\alpha} \prec \pi_{\beta} \quad M_{\alpha}$ is in the closure of $\left\{M_{r}, \gamma \in \operatorname{supp} \mu \backslash\{0\}\right\}$ in $G$-Max $C^{*}(N)$ with respect to hull-kernel topology, therefore $\alpha \in \operatorname{supp} \mu$.

THEOREM 3.7. Suppose $G \in[\mathrm{SIN}]$ and let $H$ be a closed subgroup of $G$ contained in $G_{F}$. If $\psi \in \hat{H}$, and $\pi \in \hat{G}$ is weakly contained in ${ }_{G} U^{\psi}$ then $\pi \mid H$ weakly contains $\psi$.

Proof. By [7, Thm. 2.11; 16, Lemma 4.3] any SIN-group $G$ is a projective limit of Lie groups $G / K_{j}, j \in J, K_{j}$ compact normal. In particular, every $G / K_{j}$ is first countable. By Proposition 2.3 in [16], there exists $j \in J$ such that $\pi\left(K_{j}\right)=\{I\}$. Since $K_{j} H / K_{j}$ is contained in $\left(G / K_{j}\right)_{F}$, by Proposition 2.8 we may assume $G$ to be first countable.

Now let $\psi=\pi_{\gamma}, \gamma \in P^{1}(H)$, and let $\varphi \in P^{1}(G)$ be an extension of $\gamma$ such that $\pi_{\varphi}$ is weakly equivalent to $U^{\psi}$ (such a function $\varphi$ exists by Proposition 3.2). Then

$$
\pi \prec U^{\psi} \quad \text { implies } \pi\left|G_{F} \prec \pi_{\varphi}\right| G_{F} \text {. }
$$

By (3.1) $\operatorname{ker}\left(\pi_{\varphi} \mid G_{F}\right)=\operatorname{ker} \pi_{\left(\varphi \mid G_{F}\right)}$ and there exists $\alpha \in E\left(G_{F}, G\right)$ such that $\operatorname{ker} \pi_{\alpha}=\operatorname{ker} \pi \mid G_{F}$ (see Remark 3.4). Next, take some maximal measure $\nu$ on $P_{1}\left(G_{F}\right)$ with resultant $\varphi \mid G_{F}$. By Lemma 3.6 there is $\rho \in \operatorname{supp} \nu$ with $\rho^{G}=\alpha\left(H=\{e\}, \beta=\left(\varphi \mid G_{F}\right)^{G}\right)$, therefore

$$
\operatorname{ker} \pi_{\rho}=\bigcap_{x \in G_{F}} M_{\rho x} \supseteqq \bigcap_{x \in G} M_{\rho x}=M_{\rho G}=\operatorname{ker} \pi \mid G_{F}
$$

and then

$$
\pi_{\rho}|H \prec \pi| H .
$$

As in the proof of Lemma 4.4 in [15] one shows: there exists a net $\left\{\rho_{i}\right\} \subseteq P_{1}\left(G_{F}\right)$ and $r_{i} \geqq 0, i \in I$, with

$$
r_{i}\left(\varphi \mid G_{F}\right)-\rho_{i} \in P\left(G_{F}\right)
$$

such that $\rho$ is the weak $*$-limit of $\left\{\rho_{i}\right\}$. Since

$$
\left\|\rho_{i}\right\|=\rho_{i}(e) \leqq 1 \text { and } \lim \inf \left\|\rho_{i}\right\| \geqq\|\rho\|=\rho^{\alpha}(e)=1
$$

we may assume $\rho_{i}(e)=1$. Then $\rho=\lim \rho_{i}$ uniformly on compact sets in $G$ thus $\rho\left|H=\lim \rho_{i}\right| H$. Since $\gamma$ is indecomposable and $\varphi\left|H=\gamma, r_{i} \gamma-\rho_{i}\right| H \in P(H), i \in I$, implies $\rho_{\imath} \mid H=\gamma$ therefore $\rho \mid H$ $=\gamma$. Then $\psi=\pi_{\gamma}$ is a subrepresentation of $\pi_{\rho} \mid H$ and by (3.2) $\psi \prec \pi \mid H$ follows.

REMARK. Since groups $G \in[F C]^{-} \cap[\mathrm{SIN}]$ are amenable [14] it 
follows from Theorem 3.7 that they have property RFP. For arbitrary $G \in[F C]^{-}$there exists a compact normal subgroup $K$ of $G$ such that $G / K \in[F C]^{-} \cap[\mathrm{SIN}]$ thus $G$ satisfies RFP by Corollary 2.9. This completes the proof of Theorem A.

LEMMA 3.8. Let $H$ be a closed subgroup of $G \in[\mathrm{SIN}]$ such that $H=H_{F}$ and for $\beta \in P^{1}(G, H)$ let $\nu$ be a maximal measure on $P_{1}(G, H)$ representing $\beta$. If $0 \notin \operatorname{supp} \nu$ then

$$
\operatorname{supp} \mu_{\beta \mid H}=\{\sigma \in E(H) ; \sigma=\rho \mid H, \rho \in \operatorname{supp} \nu\}
$$

in particular, $0 \notin \operatorname{supp} \mu_{\beta \mid H}$.

Proof. The restriction map from $P_{1}(G)$ into $P_{1}(H)$ is not weak *-continuous in general, but if $0 \notin \operatorname{supp} \nu$

$$
\text { supp } \nu \subseteq F(G, H) \subseteq P^{1}(G, H)
$$

therefore the map $R: \rho \rightarrow \rho \mid H$ from supp $\nu$ into $P_{1}(H, H)$ is continuous. Since $E(H)$ is closed in Pontryagin topology the image $\nu^{R}$ of $\nu$ has support

$$
R(\operatorname{supp} \nu) \subseteq R(F(G, H)) \subseteq E(H)
$$

by Lemma 3.5. By the proof of Lemma 2.9 in [9]

$$
\begin{aligned}
\beta(x) & =\int_{\text {supp } \nu} \rho(x) d \nu(\rho) \text { for } x \in G \text { thus } \\
\beta(s) & =\int_{E(H)} \gamma(s) d \nu^{R}(\gamma) \text { for } s \in H \text { and then } \\
\langle\beta \mid H, h\rangle & =\int_{P_{1}(I I, H)}\langle\gamma, h\rangle d \nu^{R}(\gamma) \text { for } h \in L^{1}(H)
\end{aligned}
$$

hence $\nu^{R}=\mu_{\beta \mid I I}$.

COROLLARY 3.9. Let $N$ be a closed normal subgroup of $G \in$ [SIN] contained in $G_{F}$ and let $\alpha \in E(N, G)$. If $F, H$ are closed subgroups of $N, F \subseteq H$, and if $\nu$ is a maximal measure on $P_{1}(H, F)$ with resultant $\alpha \mid H$ then $0 \notin \operatorname{supp} \nu$.

Proof. Let $\nu_{1}$ be a maximal measure on $P_{1}(N, H)$ with $r\left(\nu_{1}\right)=\alpha$, then $\{\alpha\}=\left(\operatorname{supp} \nu_{1}\right)^{G}$ by Lemma 3.6, therefore $0 \notin \operatorname{supp} \nu_{1}$. By Lemma $3.80 \notin \operatorname{supp} \mu_{\alpha \mid I I}$ and again by Lemma $3.60 \notin$ supp $\nu$.

REMARK. The same holds if $\alpha$ is the resultant of a probability measure $\mu$ on $P_{1}(N, G)$ with $\operatorname{supp} \mu \subseteq E(N, G)$.

G. Schlichting has pointed out to me the following corollary. 
Corollary 3.10. Let $G, N, \alpha$ as in Corollary 3.9 and let $H$ be a compact subgroup of $N$. Then $\mu_{\alpha \mid H}$ has finite support.

Proof. By [12, Satz 3], $E(H)$ is discrete and

$$
\operatorname{supp} \mu_{\alpha \mid H} \subseteq E(H) \quad \text { (Corollary 3.9) . }
$$

REMARK 3.11. Let $G \in$ [SIN] and $N \leqq G_{F}$ be a discrete normal subgroup of $G$. Since every element in $N / Z(N)$ has finite order, $Z(N)$ the center of $N$, every finite set in $N / Z(N)$ generates a finite subgroup [19, Thm. 4.3.2 and Corollary 2, p. 45]. Thus every finite subset of $N$ is contained in a normal subgroup $M$ of $G$ such that

$$
Z(N) \subseteq M \cong N \quad \text { and } \quad[M: Z(N)]<\infty
$$

THEOREM 3.12. Let $G$ be an amenable SIN-group and $H \subseteq G_{F}$ be a closed subgroup. If $\pi \in \hat{G}$, and if $\psi \in \hat{H}$ is weakly contained in $\pi \mid H$, then ${ }_{G} U^{\psi}$ weakly contains $\pi$.

Proof. Take $\alpha \in E\left(G_{F}, G\right), \sigma \in E(H)$ such that $\pi \mid G_{F}$ is weakly equivalent to $\pi_{\alpha}$ and $\psi$ is weakly equivalent to $\pi_{\sigma}$ (see Remark 3.4 and the remarks preceding Proposition 3.3). By (2.4), $\psi<\pi \mid H$ implies $\pi_{o} \prec \pi_{\alpha} \mid H \prec \pi_{\alpha \mid H I}$ therefore

$$
\sigma \in \operatorname{supp} \mu_{\alpha \mid I I} \quad \text { by Lemma 3.6. }
$$

It is sufficient to prove

$$
\pi_{\alpha} \prec\left\{\left({ }_{G_{F}} U^{\sigma}\right)^{x}, x \in G\right\} .
$$

Actually, since the representations of $G$ induced by $\left({ }_{G_{F}} U^{o}\right)^{x}, x \in G$ are equivalent to ${ }_{G} U\left({ }_{G_{F}} U^{\sigma}\right)={ }_{G} U^{\sigma}$ it follows from (3.3) and [6]

$$
\pi \prec{ }_{G} U^{\pi \mid G_{F}} \prec{ }_{G} U^{\pi_{\alpha}} \prec{ }_{G} U^{\sigma} \prec{ }_{G} U^{\psi} .
$$

Therefore let $Y$ be a compact subset of $G_{F}$. By [22] there exist normal subgroups $V, L$, and $K$ of $G$ such that $V$ is a vector group, $K$ is compact open in $L, L / K \subseteq(G / K)_{F}$ and $G_{F}=V L$ is a direct product of $V$ and $L^{3}$. Then by Remark 3.11 we can choose normal subgroups $M, Z$ of $G, K \subseteq Z \subseteq M \leqq L$, such that $[M: Z]<\infty, Z / K$ is the centre of $L / K$ and $Y$ is contained in $N=V M . \quad V Z$ is an open subgroup as it contains $V K$. Now we consider the chain of subgroups

$$
H \subseteq H K \cong H V Z \subseteq H N .
$$

\footnotetext{
${ }^{3}$ See the footnote to the proof of Theorem 2.7.
} 
Since SIN-groups are unimodular $H K / H$ and $H N / H V Z$ have finite volume. $H K$ is normal in $H V Z$ as $Z / K$ is the centre of $L / K$ and $V$ is central in $G_{F}$. Therefore by Corollary 2.12

$$
\pi_{\rho} \prec{ }_{H N} U^{\rho \mid H} \text { for } \rho \in P(H N) \text {. }
$$

Now let $\nu$ be a maximal measure on $P_{1}(H N, H)$ with resultant $\alpha_{\mathrm{a}} \mid H N$. By Corollary 3.9 and Lemma 3.8, there exists $\rho \in \operatorname{supp} \nu$ such that

$$
\rho \mid H=\sigma .
$$

Since $\alpha \mid H N$ is a class function on $H N \rho^{H N} \in \operatorname{supp} \mu_{\alpha \mid H N}$ by Lemma 3.6, thus $\pi_{\rho H N} \prec \pi_{\alpha \mid H N}$. As $\operatorname{ker} \pi_{\rho}=\operatorname{ker} \pi_{\rho H N}$ we get $\pi_{\rho} \prec \pi_{\alpha \mid H N}$, and $\pi_{\rho} \prec{ }_{H N} U^{o}$ follows from (3.4). Since $H N$ is open in $G_{F}$ we obtain by inducing up to $G_{F}$

$$
\pi_{\varphi} \prec \pi_{\beta} \text { and } \pi_{\varphi} \prec{ }_{a_{F}} U^{o}
$$

where $\varphi \in P\left(G_{F}\right)$ and $\beta \in P\left(G_{F}\right)$, respectively, denote the trivial extensions of $\rho$ and $\alpha \mid H N, \varphi(x)=0=\beta(x)$ if $x \notin H N$. Since $\pi_{\varphi \sigma}$ is weakly equivalent to $\left\{\left(\pi_{\varphi}\right)^{x}, x \in G\right\}$ therefore

$$
\pi_{\varphi^{G}} \prec \pi_{\beta G} \text { and } \pi_{\varphi^{G}} \prec\left\{\left(_{G_{F}} U^{o}\right)^{x} ; x \in G\right\} .
$$

Finally, take $\gamma \in E\left(G_{F}, G\right)$ such that $\pi_{\gamma} \prec \pi_{\varphi^{G}}$, then

$$
\pi_{\gamma \mid N} \prec \pi_{\beta G_{\mid N}}
$$

But if $B=\overline{I(N, G)}$ and $n \in N$

$$
\beta^{\alpha}(n)=\int_{B} \beta\left(\tau^{-1}(n)\right) d \tau=\int_{B} \alpha\left(\tau^{-1}(n)\right) d \tau=\alpha(n)
$$

therefore $M_{\gamma \mid N} \supseteqq M_{\alpha \mid N}$. Since $E(N, G)$ is homeomorphic to $G$-Max $C^{*}(N)$ and $\gamma|N, \alpha| N \in E(N, G)$

$$
\gamma|N=\alpha| N
$$

thus $\gamma$ and $\alpha$ agree on $Y$ and $\pi_{\gamma} \prec\left\{\left({ }_{G_{F}} U^{o}\right)^{x} ; x \in G\right\}$ consequently

$$
\pi_{\alpha} \prec\left\{\left({ }_{G_{F}} U^{o}\right)^{x} ; x \in G\right\} \text {. }
$$

REMARK. Theorem B follows from Theorem 3.7 and Theorem 3.12 .

COROLLARY 3.13. For SIN-groups $G$ the following conditions are equivalent

1. $G \in[F P]$

2. $G \in[R F P]$

3. $G=G_{F}$. 


\section{Proof. Clearly, $1 \Rightarrow 2,2 \Rightarrow 3$ by Theorem 2.7 and $3 \Rightarrow 1$ follows from Theorem $B$.}

\section{REFERENCES}

1. R. J. Blattner, Positive definite measures, Proc. Amer. Math. Soc., 14 (1963), 423428.

2. J. Dixmier, Les $C^{*}$-algèbres et leurs représentations, Paris: Gauthier-Villars 1964.

3. R. Felix, R. W. Henrichs and H. L. Skudlarek, Topological Frobenius reciprocity for projective limits of Lie groups, Math. Z., 165 (1978), 19-28.

4. J. M. G. Fell, Weak containment and induced representation of groups $I I$, Trans. Amer. Math. Soc., 110 (1964), 424-447.

5. E. C. Gootman, Weak containment and weak Frobenius reciprocity, Proc. Amer. Math. Soc., 54 (1976), 417-422.

6. F. P. Greenleaf, Amenable actions of locally compact groups, J. Functional Analysis, 4 (1969), 295-315.

7. S. Grosser and M. Moskowitz, Compactness conditions in topological groups, J. Reine Angew. Math., 246 (1971), 1-40.

8. R. W. Henrichs, Die Frobeniuseigenschaft FP für diskrete Gruppen, Math. Z., 147 (1976), 191-199.

9. - Über Fortsetzung positiv definiter Funktionen, Math. Ann., 232 (1978), 131-150.

10. E. Hewitt and K. A. Ross, Abstract Harmonic Analysis I, Berlin-Heidelberg-New York, Springer 1963.

11. A. Hulanicki and T. Pytlik, On cyclic vectors of induced representations, Proc. Amer. Math. Soc., 31 (1972), 633-634.

12. E. Kaniuth, Zur harmonischen Analyse klassenkompakter Gruppen, Math. Z., 110 (1969), 297-305.

13. - Topology in duals of SIN-groups, Math. Z., 134 (1973), 67-80.

14. H. Leptin, Zur harmonischen Analyse klassenkompakter Gruppen, Inventiones Math., 5 (1968), 249-254.

15. J. R. Liukkonen, Dual spaces of locally compact groups with precompact conjugacy classes, Trans. Amer. Math. Soc., 180 (1973), 85-108.

16. C. C. Moore, Groups with finite dimensional irreducible representations, Trans. Amer. Math. Soc., 166 (1972), 401-410.

17. R. Mosak, The $L^{1-}$ and $C^{*}$-algebras of $[F I A]_{B}$-groups and their representations, Trans. Amer. Math. Soc., 163 (1972), 277-310.

18. M. A. Rieffel, Induced representations of $C^{*}$-algebras, Advances Math., 13 (1974), 167-257.

19. D. J. S. Robinson, Finiteness Conditions and Generalized Soluble Groups I, BerlinHeidelberg-New York, Springer 1972.

20. E. Thoma, Über unitäre Darstellungen abzählbarer, diskreter Gruppen, Math. Ann., 153 (1964), 111-138.

21. Zur harmonischen Analyse klassenfiniter Gruppen, Inventiones Math., 3 (1967), 20-42.

22. T. W. Wilcox, A note on groups with relatively compact conjugacy classes, Proc. Amer. Math. Soc., 42 (1974), 326-329.

Received December 1, 1977 and in revised form October 16, 1978. This paper contains the second part of the authors Habilitationsschrift (München 1976) as well as further results on weak containment for unitary representations.

TECHNISCHE UNIVERSITÄT MÜNCHEN

8000 MÜNCHEN 2

Federal Republic of Germany 


\section{PACIFIC JOURNAL OF MATHEMATICS}

\section{EDITORS}

Donald BABBITT (Managing Editor)

University of California

Los Angeles, CA 90024

HUGo RossI

University of Utah

Salt Lake City, UT 84112

C. C. MOORE and ANDREW OGG

University of California

Berkeley, CA 94720

\section{J. DuGUNDJI}

Department of Mathematics

University of Southern California

Los Angeles, CA 90007

R. FINN and J. Milgram

Stanford University

Stanford, CA 94305

ASSOCIATE EDITORS
E. F. BECKENBACH
B. H. NeumanN
F. WOLF
K. YoSHIDA

\section{SUPPORTING INSTITUTIONS}

\author{
UNIVERSITY OF BRITISH COLUMBIA \\ CALIFORNIA INSTITUTE OF TECHNOLOGY \\ UNIVERSITY OF CALIFORNIA \\ MONTANA STATE UNIVERSITY \\ UNIVERSITY OF NEVADA, RENO \\ NEW MEXICO STATE UNIVERSITY \\ OREGON STATE UNIVERSITY \\ UNIVERSITY OF OREGON
}

\author{
UNIVERSITY OF SOUTHERN CALIFORNIA \\ STANFORD UNIVERSITY \\ UNIVERSITY OF HAWAII \\ UNIVERSITY OF TOKYO \\ UNIVERSITY OF UTAH \\ WASHINGTON STATE UNIVERSITY \\ UNIVERSITY OF WASHINGTON
}

The Supporting Institutions listed above contribute to the cost of publication of this Journal, but they are not owners or publishers and have no responsibility for its content or policies.

Mathematical papers intended for publication in the Pacific Journal of Mathematics should be in typed form or offset-reproduced, (not dittoed), double spaced with large margins. Please do not use built up fractions in the text of the manuscript. However, you may use them in the displayed equations. Underline Greek letters in red, German in green, and script in blue. The first paragraph or two must be capable of being used separately as a synopsis of the entire paper. Please propose a heading for the odd numbered pages of less than 35 characters. Manuscripts, in triplicate, may be sent to any one of the editors. Please classify according to the scheme of Math. Reviews, Index to Vol. 39. Supply name and address of author to whom proofs should be sent. All other communications should be addressed to the managing editor, or Elaine Barth, University of California, Los Angeles, California, 90024.

50 reprints to each author are provided free for each article, only if page charges have been substantially paid. Additional copies may be obtained at cost in multiples of 50 .

The Pacific Journal of Mathematics is issued monthly as of January 1966. Regular subscription rate: $\$ 84.00$ a year (6 Vols., 12 issues). Special rate: $\$ 42.00$ a year to individual members of supporting institutions.

Subscriptions, orders for numbers issued in the last three calendar years, and changes of address should be sent to Pacific Journal of Mathematics, P.O. Box 969, Carmel Valley, CA 93924, U.S.A. Older back numbers obtainable from Kraus Periodicals Co., Route 100, Millwood, NY 10546.

PUBLISHED BY PACIFIC JOURNAL OF MATHEMATICS, A NON-PROFIT CORPORATION

Printed at Kokusai Bunken Insatsusha (International Academic Printing Co., Ltd.). 8-8, 3-chome, Takadanobaba, Shinjuku-ku, Tokyo 160, Japan.

Copyright (C) 1979 by Pacific Journal of Mathematics Manufactured and first issued in Japan 


\section{Pacific Journal of Mathematics}

\section{Vol. 82 , No. 2 \\ February, 1979}

Krishnaswami Alladi and Paul Erdős, On the asymptotic behavior of large prime

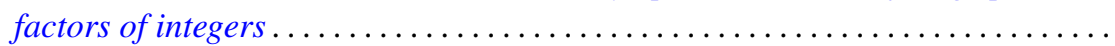

Alfred David Andrew, A remark on generalized Haar systems in $L_{p}$,

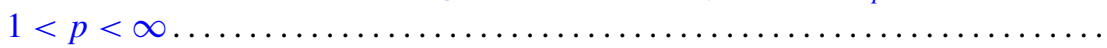

John M. Baker, A note on compact operators which attain their norm . . ........

Jonathan Borwein, Weak local supportability and applications to

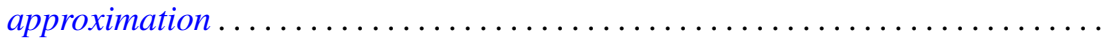

Tae Ho Choe and Young Soo Park, Wallman's type order compactification ........

Susanne Dierolf and Ulrich Schwanengel, Examples of locally compact

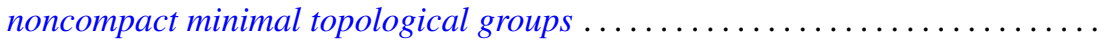

Michael Freedman, A converse to (Milnor-Kervaire theorem) $\times R$ etc. . . . . . . .

George Golightly, Graph-dense linear transformations ..................

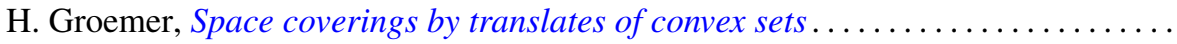

Rolf Wim Henrichs, Weak Frobenius reciprocity and compactness conditions in

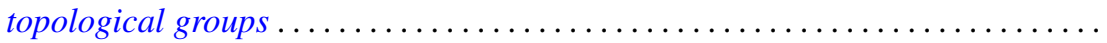

Horst Herrlich and George Edison Strecker, Semi-universal maps and universal

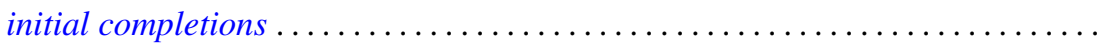

Sigmund Nyrop Hudson, On the topology and geometry of arcwise connected,

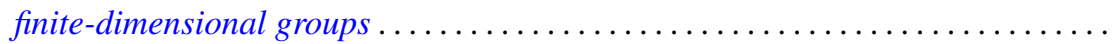

K. John and Václav E. Zizler, On extension of rotund norms. II .............

Russell Allan Johnson, Existence of a strong lifting commuting group of transformations. II.

Bjarni Jónsson and Ivan Rival, Lattice varieties covering the smallest nonmodular variety

Grigori Abramovich Kolesnik, On the order of Dirichlet L-functions .

Robert Allen Liebler and Jay Edward Yellen, In search of nonsolvable groups of

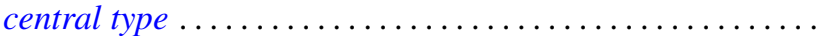

Wilfrido Martínez T. and Adalberto Garcia-Maynez Cervantes, Unicoherent plane Peano sets are $\sigma$-unicoherent ...

M. A. McKiernan, General Pexider equations. I. Existence of injective

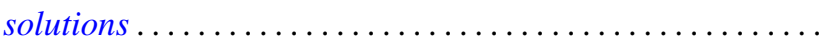

M. A. McKiernan, General Pexider equations. II. An application of the theory of webs.

Jan K. Pachl, Measures as functionals on uniformly continuous functions . .

Lee Albert Rubel, Convolution cut-down in some radical convolution algebras ...

Peter John Slater and William Yslas Vélez, Permutations of the positive integers

with restrictions on the sequence of differences. II . . .

Raymond Earl Smithson, A common fixed point theorem for nested spaces ....

Indulata Sukla, Generalization of a theorem of McFadden .... . . .

Jun-ichi Tanaka, A certain class of total variation measures of analytic measures.

Kalathoor Varadarajan, Modules with supplements .............. 